\title{
Correct techniques for extracellular recordings of electrical activity in gastrointestinal muscle
}

\section{Gregory O'Grady, Niranchan Paskaranandavadivel, Peng Du, Timothy Angeli, Jonathan C. Erickson and Leo K. Cheng}

In their Perspectives (Problems with extracellular recording of electrical activity in gastrointestinal muscle. Nat. Rev. Gastroenterol. Hepatol. 13, 731-741; 2016), Sanders et al. ${ }^{1}$ expand on previous claims that the gastrointestinal extracellular literature, together with related electrophysiology models, could be unreliable owing to contamination with movement artefacts. The essence of their claims seems to be that extracellular methods might not provide physiologically meaningful or mechanistically useful information. We feel that the authors are incorrect and mispresent our work and other competing evidence. Similar previous claims have already been evaluated and disputed in previous research from our laboratory ${ }^{2-4}$, and the reported concerns with extracellular recordings might have arisen simply owing to an incorrect application of extracellular techniques and misunderstanding of basic extracellular principles. Here, we clarify correct approaches to extracellular recordings.

Sanders et al. ${ }^{1}$ performed their extracellular recordings in vitro on devitalised tissues; however, they have published that their tissue isolation process aberrantly elevates slow-wave frequencies, causing loss of intrinsic frequency gradient $s^{5,6}$. Intrinsic frequency gradients are critical for slow-wave entrainment and generation of extracellular field potentials ${ }^{2,4}$, and extracellular data cannot be recorded in their absence ${ }^{7}$. Extrapolating findings from devitalised tissue studies to all extracellular studies is inappropriate in this context.

Furthermore, we feel that Sanders et al. ${ }^{1}$ have misrepresented basic extracellular physiology. Their representation of weak sharply oscillating biopotentials as extracellular potentials is misleading because these gastric signals do not resemble legitimate biphasic slow-wave data recorded by many research groups over a century. In their experimental studies, Sanders et al. ${ }^{1}$ seem to have recorded movement artefacts and have then attributed problems to extracellular methods in general, rather than technical issues ${ }^{8}$.

A simple validation for the morphology of gastrointestinal extracellular slow-wave potentials is achieved by performing suction and conventional contact recordings simultaneously (side-by-side) in vivo (FIG. 1). In accordance with known biophysical principles, suction extracellular recordings give a monophasic potential approximating the transmembrane potential, whereas contact electrodes give a biphasic potential ${ }^{2}$. This biphasic potential coincides with the activation phase of the monophasic potential (FIG. 1); it is upgoing before arrival of the wavefront, steeply negative when the wavefront is under the electrode, then returns to baseline. This biphasic potential configuration has been repeatedly shown over the past century to precede contractions, and, therefore, cannot be a contraction artefact ${ }^{4}$. This signal of interest is not present in extracellular data currently offered by Sanders and colleagues ${ }^{1,8}$ and must be present in the raw signal traces, with minimal filtering used only to aid interpretation ${ }^{3}$ (FIG. 2a,b). The $3-100 \mathrm{~Hz}$ bandpass filter that Sanders et al. ${ }^{1}$ advocate would grossly distort true slow-wave data because it eliminates
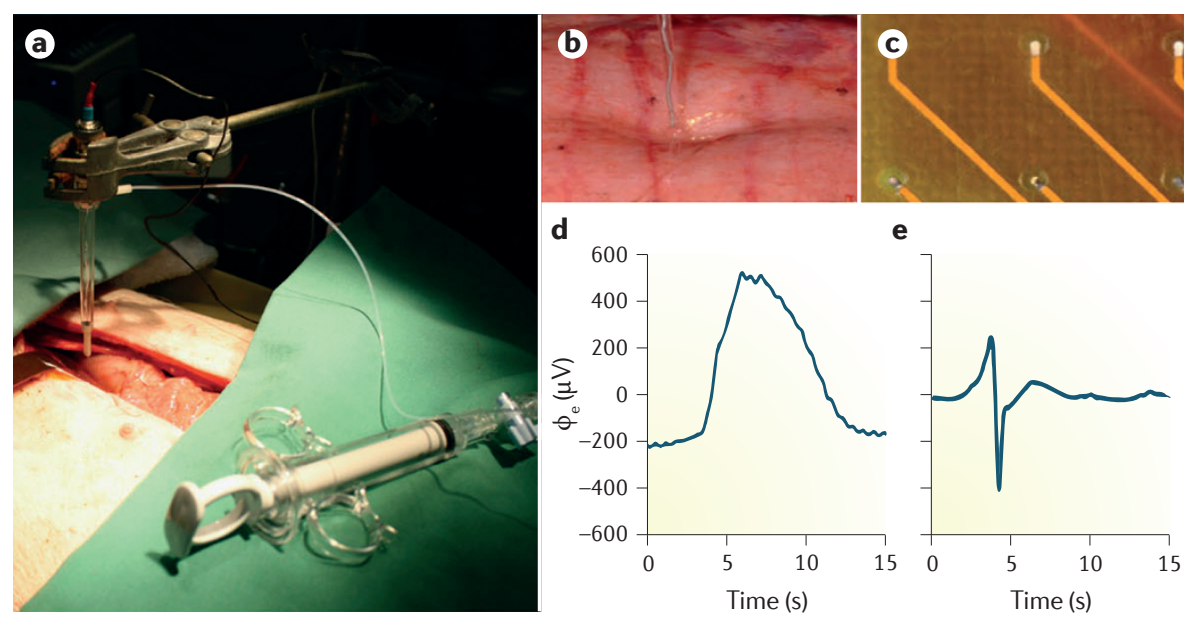

Figure 1 | Extracellular morphologies. a |Comparison of extracellular morphologies from a suction electrode and conventional serosal contact electrode employed simultaneously in vivo on adjacent regions of porcine gastric serosa. $\mathbf{b}$ |Suction electrode position. $\mathbf{c}$ |Serosal contact electrodes used for the comparison (flexible printed circuit board array). d,e | Comparison of experimental slow-wave data recorded by the two extracellular modes simultaneously. The suction electrode generates a monophasic potential, whereas the contact electrode generates a biphasic potential corresponding with the upstroke (activation / wavefront) phase of the monophasic potential. Reproduced with permission from Wiley (C) Angeli et al.J. Physiol. 591, 4567-4579 (2013). 


\section{CORRESPONDENCE}

We conclusively demonstrated that extracellular slow waves are readily recordable in vivo even during complete motion suppression by intra-arterial nifedipine administration $^{2}$. In this Perspectives, the authors ${ }^{1}$ misrepresented our methodology, incorrectly claiming we only assessed longitudinal tissue motion, undermining our validation study ${ }^{2}$. However, our intestinal segments were not arranged in straight lines; we captured curved intestinal segments within each measured field, recording motion to single-pixel (submillimetre) resolution. There was no motion. The correct interpretation is that extracellular recordings are valid when performed and analysed correctly, and routine motion suppression is not required in vivo.

We disagree with the conclusions made by Sanders et al. ${ }^{1}$ in their Perspectives. The 'problems' they describe are easily overcome if correct extracellular techniques are used, and routine motion suppression is unnecessary in vivo. Indeed, the role of extracellular methods is currently expanding, as highresolution electrical mapping is now contributing to substantial translational advances in human motility disorders ${ }^{10}$.

Gregory O'Grady is at the Auckland Bioengineering Institute, University of Auckland, Auckland 1142, New Zealand; and at the Department of Surgery, Private Bag 92019, University of Auckland, Auckland 1142, New Zealand.

Niranchan Paskaranandavadivel, Peng Du, Timothy Angeli and Leo K. Cheng are at the Auckland Bioengineering Institute, University of Auckland, Auckland 1142, New Zealand.

Jonathan C. Erickson is at the Auckland Bioengineering Institute, University of Auckland Auckland 1142, New Zealand; and at the Department of Physics and Engineering, Washington and Lee University, 204W Washington Street, Lexington, Virginia 24450, USA. a Baseline removed signals

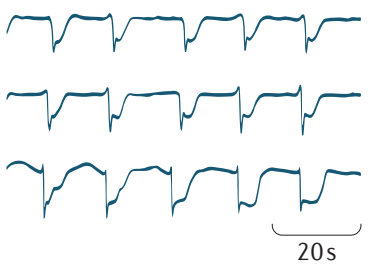

b Low pass filter at $2 \mathrm{~Hz}$ (Butterworth)

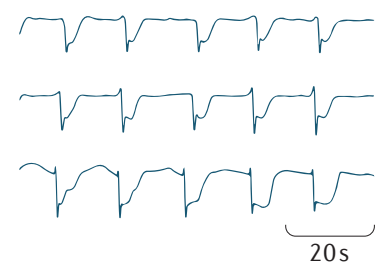

C Bandpass filter at $3-100 \mathrm{~Hz}$ (Butterworth)
Figure 2 | Comparison of filter effects on gastric serosal slow-wave signals (porcine data). a Slow-wave signals from adjacent channels sampled at $512 \mathrm{~Hz}$, with only the baseline wander removed (moving median window of $20 \mathrm{~s}$ ). $\mathbf{b}$ | The same data following application of a Butterworth $2 \mathrm{~Hz}$ low-pass filter ${ }^{3}$. c|The same data following application of a 3-100 Hz band-pass Butterworth filter as advocated by Sanders et al. ${ }^{1}$. True slow-wave data would be eliminated with this filter.
Correspondence to G.O'G. greg.ogrady@auckland.ac.nz

doi: $10.1038 /$ nrgastro.2017.15

Published online 30 Mar 2017

1. Sanders, K., Ward, S. M. \& Hennig, G. Problems with extracellular recordings of electrical activity in gastrointestinal muscle. Nat. Rev. Gastroenterol. Hepatol. 13, 731-741 (2016).

2. Angeli, T. R. et al. The bioelectrical basis and validity of gastrointestinal extracellular slow wave recordings. J. Physiol. 591, 4567-4579 (2013).

3. Paskaranandavadivel, N., O'Grady, G., Du, P. $\&$ Cheng, L. K. Comparison of filtering methods for extracellular gastric slow wave recordings. Neurogastroenterol. Motil. 25, 79-83 (2013).

4. O'Grady, G. Gastrointestinal extracellular electrical recordings: fact or artifact? Neurogastroenterol. Motil. 24, 1-6 (2012).

5. Rhee, P. L. et al. Analysis of pacemaker activity in the human stomach. J. Physiol. 589, 6105-6118 (2011).

6. O'Grady, G., Pullan, A. J. \& Cheng, L. K. The analysis of human gastric pacemaker activity. J. Physiol. 590, 1299-1300 (2012).

7. Xue, S., Valdez, D. T., Tremblay, L., Collman, P. I. \& Diamant, N. E. Electrical slow wave activity of the cat stomach: its frequency gradient and the effect of indomethacin. Neurogastroenterol. Motil. 7 157-167 (1995).

8. Bayguinov, O., Hennig, G. W. \& Sanders, K. M. Movement artifacts may contaminate extracellular electrical recordings from $\mathrm{GI}$ muscles. Neurogastroenterol. Motil. 23, 1029-e498 (2011).

9. O'Grady, G. et al. Rapid high-amplitude circumferential slow wave conduction during normal gastric pacemaking and dysrhythmia. Neurogastroenterol. Motil. 24, e299-e312 (2012).

10. Angeli, T. R. et al. Loss of interstitial cells of Cajal and patterns of gastric dysrhythmia in patients with chronic unexplained nausea and vomiting Gastroenterology 149, 56-66.e5 (2015).

\section{Acknowledgements}

The authors are funded by the New Zealand Health Research Council, the US NIH (R01 DK 64776), the NZ MedTech CoRE, the Auckland Medical Research Foundation (TA) and a Rutherford Discovery Fellowship (PD).

\section{Competing interests statement}

The authors hold grants and intellectual property applications in the field of gastrointestinal electrophysiology, and are shareholders in FlexiMap Ltd. 